\title{
Obstructive sleep apnea in patients with interstitial lung diseases: past and future
}

\author{
Charalampos Mermigkis • Izolde Bouloukaki • \\ Sophia E. Schiza
}

Received: 27 January 2013 / Accepted: 22 March 2013 /Published online: 12 April 2013

(C) Springer-Verlag Berlin Heidelberg 2013

In their article published in this issue of Sleep and Breathing, Pihtili et al. [1] present polysomnographic data in patients with interstitial lung diseases (ILDs) including 17 patients with idiopathic pulmonary fibrosis (IPF), 15 patients with stage II-III sarcoidosis, and 18 patients with pulmonary fibrosis due to scleroderma. The study revealed a high prevalence, namely $68 \%$, of obstructive sleep apnea (OSA) in this population. The prevalence of OSA was $82.3 \%$ in the IPF patients, $66.6 \%$ in the sarcoidosis patients, and $55.5 \%$ in the scleroderma patients. The OSA severity was mostly mild, while the scored respiratory events were predominantly hypopneas. An interesting feature of this study is that this increased incidence of OSA was observed after the exclusion of patients with well-known predisposing factors for OSA such as obesity and/or significant upper airway pathologies causing obstruction. The authors used a disease severity index consisting of body mass index, carbon monoxide diffusion capacity, the Modified Medical Research Council dyspnea scale, and the 6-min walking distance, with scores ranging between 0 and 10 . The OSA diagnosis rate was higher in the patients who had severity index $\geq 3$.

C. Mermigkis

Sleep Disorders Center, 401 General Army Hospital, Athens, Greece

C. Mermigkis $\cdot$ I. Bouloukaki $\cdot$ S. E. Schiza

Sleep Disorders Unit, Department of Thoracic Medicine, University General Hospital, Medical School of the University of Crete, Crete, Greece

C. Mermigkis $(\bowtie)$

Thrakis 61A, Vrilissia,

Athens, Greece

e-mail: mermigh@gmail.com
The frequency and consequences of OSA in patients with interstitial lung diseases is a field of growing research over the last years, although the medical literature on this area still remains quite limited with significant contraries between the results of older and more recent studies [2]. Prior polysomnographic studies in the mid-1980s demonstrated alterations in sleep architecture and oxygen desaturation, especially during REM sleep, but not OSA, even in morbidly obese patients with ILDs [3, 4]. The results of the study by Pihtili et al. related to IPF patients are similar to recent studies that report increased incidence of OSA and pointed the need for high clinical suspicion for possible underlying OSA in this population [5-8]. Prior studies dating back 10-20 years probably underestimated hypopneas by using only thermal sensors and not nasal pressure transducers which are recognized as the sensitive devices for hypopnea detection that represents the majority of the observed respiratory events in IPF patients based on the newer studies. Related to sarcoidosis, Pihtili et al. report significantly higher percentages of OSA compared to previous studies [9] while the only one published study in scleroderma [10] found no OSA contrary to the high percentages observed by Pihtili et al.

The available data of the above-mentioned studies show that OSA is common in patients with ILDs, especially those with IPF. Restrictive pulmonary diseases are characterized by decreased lung volumes that can reduce the upper airway stability and increase resistance due to a decreased traction on the upper airway. These changes can facilitate the upper airway collapse, especially during REM sleep when functional residual capacity is further reduced due to the inactivity of the intercostal muscles.

The morbidity and mortality of OSA are high especially when it occurs concomitantly with other respiratory diseases. Timely diagnosis and management of sleep disorders in ILDs may be an intervention that can significantly impact 
the outcomes of these patients. Especially in diseases like IPF with a poor outcome and no available effective treatment, the early diagnosis of OSA should be a primary goal. Polysomnography (PSG) is an expensive procedure and cannot be performed in all ILD patients. On the other hand, physicians are treating patients and not numbers and should not defer an interview by a sleep specialist and/or a nocturnal oximetry [11] in order to recognize the ILD patients who need a thorough evaluation with overnight PSG.

The main issue in sleep medicine is not only to recognize sleep disorders but to offer effective treatment. The only data of effective CPAP treatment are available in IPF patients with OSA. This population has a high incidence of CPAP nonacceptance or poor compliance, which can only be eliminated through an intense follow-up by a wellorganized sleep center [12]. Although it is worth to overcome such difficulties since recent data show that effective CPAP treatment in IPF patients with OSA results in a significant improvement in daily living activities based on the Functional Outcomes in Sleep Questionnaire, namely an OSA specific follow-up instrument [13].

Further studies including increased number of ILD patients are necessary to assess not only the negative influence of untreated OSA on quality of life but also the possible contribution of OSA to ILD-related morbidity and mortality.

Conflict of interest The authors declare they have no conflict of interest.

\section{References}

1. Pihtili A, Bingol Z, Kiyan E, Cuhadaroglu C, Issever H, Gulbaran Z. Obstructive sleep apnea is common in patients with interstitial lung disease (2013). Sleep Breath. doi:10.1007/s11325-013-0834-3
2. Agarwal S, Richardson B, Krishnan V, Schneider H, Collop NA, Danoff SK (2009) Interstitial lung disease and sleep: what is known? Sleep Med 10:947-951

3. Perez-Padilla R, West P, Lertzman M, Kryger M (1985) Breathing during sleep in patients with interstitial lung disease. Am Rev Respir Dis 132:224-229

4. Bye P, Issa F, Berthon-Jones M, Sullivan C (1984) Studies of oxygenation during sleep in patients with interstitial lung disease. Am Rev Respir Dis 129:27-32

5. Mermigkis C, Stagaki E, Tryfon S, Schiza S, Amfilochiou A, Polychronopoulos V, Panagou P, Galanis N, Kallianos A, Mermigkis D, Kopanakis A, Varouchakis G, Kapsimalis F, Bouros D (2010) How common is sleep-disordered breathing in patients with idiopathic pulmonary fibrosis? Sleep Breath 14:387390

6. Ruhle KH, Mermigkis C (2010) Commentary on How common is sleep-disordered breathing in patients with idiopathic pulmonary fibrosis? Sleep Breath 14:289

7. Mermigkis C, Chapman J, Golish J, Mermigkis D, Budur K, Kopanakis A, Polychronopoulos V, Burgess R, FoldvarySchaefer N (2007) Sleep-related breathing disorders in patients with idiopathic pulmonary fibrosis. Lung 185:173-178

8. Lancaster LH, Mason WR, Parnell JA, Rice TW, Loyd JE, Milstone AP, Collard HR, Malow BA (2009) Obstructive sleep apnea is common in idiopathic pulmonary fibrosis. Chest 136:772-778

9. Turner GA, Lower EE, Corser BC, Gunther KL, Baughman RP (1997) Sleep apnea in sarcoidosis. Sarcoidosis Vasc Diffuse Lung Dis 14:61-64

10. Prado GF, Allen RP, Trevisani VM, Toscano VG, Earley CJ (2002) Sleep disruption in systemic sclerosis (scleroderma) patients: clinical and polysomnographic findings. Sleep Med 3:341-345

11. Pitsiou G, Bagalas V, Boutou A, Stanopoulos I, ArgyropoulouPataka P (2012) Should we routinely screen patients with idiopathic pulmonary fibrosis for nocturnal hypoxemia? Sleep Breath. doi:10.1007/s11325-012-0716-0

12. Mermigkis C, Mermigkis D, Varouchakis G, Schiza S (2012) CPAP treatment in patients with idiopathic pulmonary fibrosis and obstructive sleep apnea-therapeutic difficulties and dilemmas. Sleep Breath 16:1-3

13. Mermigkis Ch.,Bouloukaki I, Antoniou K, Mermigkis D, Psathakis K, Giannarakis I,Varouchakis G, Siafakas N, Schiza S (2013) CPAP therapy in patients with idiopathic pulmonary fibrosis and obstructive sleep apnea. Does it offer a better quality of life and sleep? Sleep and Breathing. doi:10.1007/s11325-013-0813-8 